\title{
Living well with dementia groups: changes in participant and therapist verbal behaviour
}

\section{Richard Cheston, Ann Marshall, Angharad Jones, John Spreadbury \& Peter Coleman}

To cite this article: Richard Cheston, Ann Marshall, Angharad Jones, John Spreadbury \& Peter Coleman (2016): Living well with dementia groups: changes in participant and therapist verbal behaviour, Aging \& Mental Health, DOI: 10.1080/13607863.2016.1231171

To link to this article: http://dx.doi.org/10.1080/13607863.2016.1231171

Published online: 27 Sep 2016.

Submit your article to this journal $\sqsubset$

a

View related articles $₫$

View Crossmark data ¿ 


\title{
Living well with dementia groups: changes in participant and therapist verbal behaviour
}

\author{
Richard Cheston ${ }^{\mathrm{a}}$, Ann Marshall ${ }^{\mathrm{b}}$, Angharad Jones ${ }^{\mathrm{c}}$, John Spreadbury ${ }^{\mathrm{d}}$ and Peter Coleman ${ }^{\mathrm{e}}$ \\ aHealth and Social Sciences, University of the West of England, Bristol, England; 'bouthern Health Foundation Trust, Newtown House, Eastleigh,

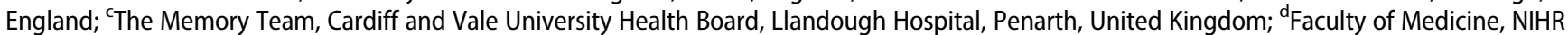 \\ CLAHRC Wessex, University of Southampton, Southampton, England; ' $F$ Faculty of Social \& Human Sciences, University of Southampton, \\ Southampton, England
}

\begin{abstract}
Objectives: This paper reports two related analyses of verbal material from seven Living Well with Dementia groups: the first examines changes in the verbal behaviours of participants across the course of the sessions in all seven groups; while the second contrasts therapist behaviour in two groups.

Methods: In the first analysis, recordings of three sessions from each group were transcribed and participant descriptions of dementia were analysed using the Markers of Assimilation of Problematic Experiences of Dementia (MAPED) rating procedure. In the second analysis, therapist behaviour in weeks 2 and 8 from two groups ( $F$ and $G$ ) was analysed using the Hill Counsellor Verbal response rating scale. Inter-rater reliabilities for the two sets of ratings were 'good' and 'very good', respectively. Results: For the MAPED ratings, a five by four contingency table was analysed using chi-squared, which indicated a highly significant change in assimilation. There were significant higher levels of level 1 and 2 markers in the first two sessions and level 4 for sessions 5 and 6 . Facilitators used significantly more direct guidance and information giving behaviour in the second session at Location F compared to Location G.

Conclusions: The results suggest that important changes occurred in the way that dementia was described across the seven LivDem groups: this includes both reductions in the avoidance of direct references to dementia after the first two sessions, as well as an increase in 'insight' statements. Directive facilitator behaviour may be associated with poorer outcomes.
\end{abstract}

\section{ARTICLE HISTORY}

Received 2 June 2016

Accepted 29 August 2016

\section{KEYWORDS}

Alzheimer's disease; psychotherapy; awareness; insight; dementia

\section{Introduction}

In the UK, government strategies in all four devolved countries have been to prioritise the development of robust methods of identifying people affected by dementia at an early stage in their illness (Department for Health, Social Services and Public Safety [DHSSPS], 2011; Department of Health, 2009; Scottish Government, 2010; Welsh Government, 2011). A significant factor directing this policy is the belief that early diagnosis facilitates people with dementia being able to plan ahead and to take control of their illness. This, in turn, is thought to improve long-term quality of life and to reduce care-giver strain. However, despite this emphasis on early detection, there are widespread concerns that not enough support is provided to people affected by dementia and their families once they have received a diagnosis (Watts, Cheston, Moniz-Cook, Burley, \& Guss, 2014). This is especially problematic for the significant number of people diagnosed with dementias such as vascular dementia, for whom prescribing of cholinesterase inhibitors is not an option and where non-pharmacological approaches are the only available treatments.

Living Well with Dementia groups. The Living Well with Dementia (or LivDem) group therapy intervention focuses on enabling the person affected by dementia to adapt to their illness. The intervention incorporates elements of psychotherapy (e.g. a focus on encouraging participants to share feelings associated with dementia such as embarrassment, worry and sadness) and psycho-education, including information about memory loss, dementia and medical treatments. The content of the 10 sessions has been standardised into a treatment manual. ${ }^{1}$

In a pilot study comparing the impact of LivDem to a waiting list control condition, seven groups were established. All seven groups were led by staff working within National Health Service (NHS) memory clinics from a range of professional backgrounds (five were occupational therapists, four were psychiatric nurses, three were nursing support workers with the remaining two facilitators being a psychology assistant and a trainee clinical psychologist). Prior to working on the project, facilitators' experiences of group work were also varied, with over half having no previous experience of working in a group context. All the facilitators attended a two-day training course, and received supervision from clinical psychologists at least three times during the course of the intervention.

The group consisted of eight weekly sessions of 75 minutes. Groups had between five and seven participants, all of whom had dementia. Family members or friends were invited to a preliminary and concluding session, but otherwise did not attend any of the eight LivDem sessions. The content of sessions was delivered in pairs: sessions 1 and 2 set out a simple model of how memory works and were designed to facilitate participants discussing their experiences of memory loss; sessions 3 and 4 focussed on the relationship between stress and memory; weeks 5 and 6 described the different 
Table 1. Structure of Living Well with Dementia intervention.

\begin{tabular}{|c|c|c|c|}
\hline Week & Attended by & Title of session & Content \\
\hline Preliminary & Participants and carers & Welcome and introductions & $\begin{array}{l}\text { Introduction to the group and to other participants, familiarisation } \\
\text { with structure, discussion of recovery model }\end{array}$ \\
\hline 1 and 2 & Participants & Problems and frustrations with memory & $\begin{array}{l}\text { Participants identify problems associated with memory loss. } \\
\text { Description of nature of short-term memory loss - compared to } \\
\text { filing letters in a filing cabinet }\end{array}$ \\
\hline 3 and 4 & Participants & $\begin{array}{l}\text { Finding a way through feelings and coping } \\
\text { with stress }\end{array}$ & $\begin{array}{l}\text { Discussion of emotional impact of memory problems and other } \\
\text { cognitive symptoms. Discussion of the impact of anxiety on } \\
\text { memory loss - practicing relaxation }\end{array}$ \\
\hline 5 and 6 & Participants & $\begin{array}{l}\text { What is dementia? Impact of the diagnosis on } \\
\text { friends and family }\end{array}$ & $\begin{array}{l}\text { Focuses on the process and assessment and diagnosis. Information } \\
\text { about different types of dementia, treatment and choices (e.g. } \\
\text { driving). Discussion about whether to tell others about the } \\
\text { diagnosis }\end{array}$ \\
\hline 7 and 8 & Participants & Living as well as you can. Staying active & $\begin{array}{l}\text { Importance of healthy lifestyle - activity, diet and preparing for the } \\
\text { future }\end{array}$ \\
\hline Consolidation & Participants and carers & Bringing it all back together & \\
\hline
\end{tabular}

forms of dementia and issues around telling others about the diagnosis; while the final two sessions looked at practical ways to 'live well' with dementia. The pairing of sessions allowed facilitators to have some flexibility in how they delivered the content across these two sessions (see Table 1), while the pace of the sessions was intended to avoid potential problems that can arise from giving participants too much threatening information at too early a point. A DVD of people affected by dementia talking about different aspects of their illness, and which paralleled the content of the sessions could also be played during sessions at the discretion of the group facilitators.

All of the participants had received a diagnosis of probable Alzheimer's disease, vascular dementia or dementia with Lewy bodies within the previous 18 months. Participants were excluded from the study if they had a significant premorbid history of mental health problems. Sixty participants were randomly allocated to one of two conditions: the LivDem group intervention; or to a usual care control arm. The protocol was registered online (ISRCTN 25079950), ${ }^{2}$ and received approval from NRES Ethics Committee South Central -Oxford B. ${ }^{3}$ The overall attendance rate at sessions was $83 \%$. Changes in levels of outcome measures in the two arms of the pilot study have been reported elsewhere (Marshall et al., 2015). Briefly, the study met its recruitment targets, with a relatively low attrition rate for the intervention arm. As a pilot study, the trial was not powered to find statistically significant results and although there was improvement in the intervention group compared to the control group for quality of life and self-esteem, after controlling for baseline variance, these findings were not significant. However, the effect size of 0.46 is consistent with that found for other facilitator led support groups (e.g. Logsdon et al., 2010).

Alongside testing for differences in outcome measures, we have also begun to look at the process of change that occurs within the intervention. The use of process measures within psychotherapy complements more conventional outcome measures and plays an especially important role in developing new forms of psychotherapy. For instance, psychotherapy process research can help to identify therapist behaviours that either block or enhance the change process.

\section{Assimilation of problematic voices: a process model of psychotherapy change}

The assimilation model of problematic voices (e.g. HonosWebb \& Stiles, 1998; Honos-Webb, Lani, \& Stiles, 1999; Stiles,
1999, 2001; Stiles, Meshot, Anderson, \& Sloan, 1992) suggests that most experiences an individual encounters during their life can be routinely absorbed into their identity. However, some experiences are too threatening to allow an easy assimilation. When an individual is faced by such a psychologically problematic experience then, instead, elements of the experience may be initially pushed away from conscious awareness. Awareness of these problematic experiences is voiced gradually and indirectly, as the dominant voice is that of the continuity of identity. The assimilation of the problematic experience occurs through a dialogue between these two voices.

A series of qualitative studies have used an adapted form of the assimilation model (the Markers of Assimilation of Problematic Experiences of Dementia or MAPED) to examine the nature of the psychological change that is required for people to 'come to terms with' dementia. Amongst other findings, these studies have described the importance of disclosures of shame within a group (Watkins, Cheston, Jones, \& Gilliard, 2006), the reciprocal turntaking discourse of couples (Snow, Cheston, \& Smart, 2015) and the use of metaphors or stories as indirect explorations of threat (Cheston, Jones, \& Gilliard, 2004). More recently, Cheston (2016) has described how a fear of loss of internal control may act to prevent people with dementia from being able to articulate especially problematic or threatening aspects of their dementia.

\section{Process analysis of LivDem sessions}

A preliminary process analysis of one of the seven LivDem groups has suggested that the four participants in this group talked more openly about their dementia at the end of the eight, participant-only sessions compared to the beginning (Cheston, Gatting, Marshall, Spreadbury, \& Coleman, 2015). However, this preliminary study looked at only one group and did not address differences in therapist behaviour.

\section{Aims of the current study}

In this paper, we will describe two studies: in the first study, we will sample all seven locations where LivDem was implemented during the pilot trial, taking three sessions at random from each location, and use the MAPED process of analysis to identify whether there were changes in participant discourse about dementia over the course of the groups. In the second study, we will use MAPED to identify potentially important clinical differences between two locations. We will then 
examine whether there are differences in therapist verbal behaviour in these two locations.

\section{Study one: changes in participant verbal behaviour (assimilation of dementia) across sessions}

\section{Methodology}

\section{MAPED analysis}

The MAPED scale and procedure used in this study to identify markers of assimilation was adapted by Lishman, Cheston, and Smithson (2016) from the Markers of Assimilation of Problematic Voices Scale (Honos-Webb \& Stiles, 1998; HonosWebb, Surko, \& Stiles, 1998; Honos-Webb, Surko, Stiles, \& Greenberg, 1999). ${ }^{4}$ Ratings of these markers take into account both the use of language to frame dementia and the emotional intensity that surrounds this discourse (see Table 2).

Use of the MAPED scale involves reading a transcript of therapy or an interview and identifying passages in which there are specific speech markers or characteristic descriptions of dementia. Each stage or level of the Assimilation process can be identified through a number of different speech markers. For instance, a passage would be coded as falling into level 1 (unwanted thoughts) if the account included an indirect description of one aspect of dementia such as memory loss but without explicitly recognising this as being related to dementia. At the same time, the person's level of affect also indicates the presence of distress (e.g. feelings of anxiety, anger or sadness). A marker of a level 1 rating carries with it the implication that elements of dementia are being avoided because this is experienced as being frightening or destabilising to the person's emotional equilibrium. Level 1 markers include: changing the conversation; locating the existence of dementia elsewhere by talking about other people and not themselves as having dementia; limiting the problems they are experiencing; references to fears of being mad or abnormal; asserting that other areas of functioning are unimpaired or that they can be easily overcome or other speech acts that minimise the significance of the dementia experience. In contrast, a level 2 marker involves a greater recognition of acknowledgement of the problematic material in which the person appears to be in distress that appears to arise from some internal conflict relating to dementia. In subsequent levels, the person is more able to name the problematic material (level 3), making links with the past or otherwise finding some distance from the emotional intensity of the problem (level 4), working through the psychological problems caused by the problematic material (level 5) and identifying partial solutions (level 6).

Table 2. Markers of Assimilation of Problematic Experiences of Dementia (MAPED) scale (from Cheston et al., 2015).

\begin{tabular}{ll}
\hline Assimilation level & \multicolumn{1}{c}{ Content and affect } \\
\hline 0 - Warded off & $\begin{array}{c}\text { Content is un-formed and features avoidance. Minimal } \\
\text { affect, reflecting successful avoidance } \\
\text { Emergence of thoughts associated with dementia or } \\
\text { memory loss. Further discussion may be avoided and } \\
\text { dementia is likely to be talked around rather than named } \\
\text { directly. Unfocused strong emotions (e.g. anxiety, fear, } \\
\text { anger sadness) are more salient than the content }\end{array}$ \\
& $\begin{array}{l}\text { The problematic experience of dementia is acknowledged } \\
\text { and uncomfortable associated thoughts are described. } \\
\text { The person is in distress, and this seems to come from } \\
\text { some internal conflict relating to dementia, so that in } \\
\text { talking they seem to be caught up in the moment of the } \\
\text { emotion. Affect focused on acute psychological pain or } \\
\text { panic }\end{array}$
\end{tabular}

3 - Problem statement or clarification

4 - Understanding/insight

The person acknowledges the existence of dementia and is able to describe how this makes them feel, or how they react to this. They are able to stand back from their feelings. Curiosity of affect, with mixed pleasant and unpleasant recognitions

5 - Application/working through

6 - Problem solution
The person acknowledges the existence of dementia as a problem and attributes this to an illness such as dementia. Affect is negative but manageable
(1) Changing the conversation

(2) Telling a contradictory story

(3) Fear-of-loss-of-control (e.g. being mad or abnormal)

(4) Dementia is located elsewhere or referred to indirectly (e.g. as ' $i t^{\prime}$ )

(5) The significance of dementia is otherwise minimised

Expressions of

(1) Feelings of sadness, depression, worry or anxiety.

(2) Anger or irritation about some aspect of dementia

(3) Puzzlement or confusion about what is happening to them

(4) Feeling overwhelmed or that things are getting worse

(5) Stories that point to the problem but are not clearly described

(6) Use of metaphors to talk about the problem

(1) Describing their reaction to dementia, which may include mixed feelings (e.g. 'yes .... But')

(2) The person appears 'stuck' and sees no way out

(3) Developing a clearer understanding of dementia (e.g. asking questions or being curious)

(4) Describing contradictions in the illness

(5) Describing that previous difficulties in recognising the problem

(1) Describing situations where their feelings differ in intensity

(2) Emotional distance from the dementia (e.g. through use of humour)

(3) Making links between dementia and others areas of their life

(4) Making links between the past and the present

(5) Comparing themselves with others in a worse position

(1) Weighing up attempts at a partial solution to the problem (e.g. taking medication)

(2) Acknowledging deterioration and explicitly describing some acceptance

(1) Achieving a change in their life in living with the dementia that they feel positive about

(2) Change in their understanding of what is happening to them or their relationships with others

(3) Comments on how others have noticed that they have changed

(1) The person successfully uses their new solutions in new situations 
Table 3. Analysis of sessions from different group locations.

\begin{tabular}{|c|c|c|c|c|c|c|c|c|}
\hline \multirow[b]{2}{*}{ Location } & \multicolumn{8}{|c|}{ Session number } \\
\hline & 1 & 2 & 3 & 4 & 5 & 6 & 7 & 8 \\
\hline$A$ & & & & & $x$ & & $x$ & $\bar{x}$ \\
\hline B & $x$ & & $x$ & & & $x$ & & \\
\hline C & & & $x$ & $x$ & & $x$ & & \\
\hline D & $X$ & & & & $x$ & $x$ & & \\
\hline$E$ & $x$ & & $x$ & & $x$ & & & \\
\hline $\mathrm{F}$ & & $x$ & & & & & $x$ & $x$ \\
\hline G & & $\mathrm{X}$ & & $X$ & & & & $x$ \\
\hline $\begin{array}{l}\text { Total number of } \\
\text { sessions analysed }\end{array}$ & 3 & 2 & 3 & 2 & 3 & 3 & 2 & 3 \\
\hline
\end{tabular}

The process for identifying markers consists of five steps:

- Selection of sessions and transcribing. All participants in the LivDem study gave their consent for each session to be audio-recorded. Fifty-one of the 56 sessions which were solely for people with dementia across the seven different sites were recorded. For each site, three sessions were transcribed (see Table 3), which meant that for each of the eight sessions, either two or three transcriptions were available for analysis.

- Familiarisation. The initial step of the analysis involves intensive exposure to the transcripts, making systematic notes to locate passages that relate to change, cognitive loss or expressions of heightened affect.

- Extracting passages. Speech markers are identifiable events in discourse that recur throughout the transcripts and that indicate important clinical phenomena (HonosWebb et al., 1998). In this analysis, the first author identified 410 extracts in which one or more of the group participants either referred directly to an aspect of dementia or were provided with a clear opportunity to do so (e.g. through a question by a facilitator) but avoided doing so.
- Training. The third author was trained in using MAPED by the first author, which included rating 16 extracts that had originally been analysed by Lishman et al. (2016). Overall agreement level after training was $81 \%$ with a Kappa score of 0.691 (standard error $(\mathrm{SE})=0.126$ ) which is considered to be 'good'.

- Rating passages and clarifying disagreements. The third author then independently coded the extracts, with the first author independently coding those 148 extracts where confidence levels were rated as being low (a score of 3 out of 5 or less). Thirteen extracts were discarded as being unclassifiable. Of the remaining 135 extracts, there was agreement on 114, with the remaining 21 extracts being resolved through discussion. The weighted Kappa score was 0.736 , which indicates that the strength of agreement between the two raters can be considered to be 'good'.

\section{Results}

In all, ratings of 397 extracts were made (see Figure 1). Although, there are potentially eight MAPED levels, the final level (mastery) was not used as a code, while level 0 (warding off) was coded just three times, from one session only and a level 6 marker (problem solution) was identified just twice. Accordingly, markers from levels 0 and 6 were treated as outliers, and trimmed or excluded from the analysis. Sessions were grouped into pairs, reflecting the way in which they were structured during the intervention.

\section{Differences in assimilation across sessions}

A chi-squared analysis using a $5 \times 4$ contingency table showed that the interaction between MAPED stages and sessions was highly significant $\left(\chi^{2}(12)=48.109, p<0.001\right)$. In

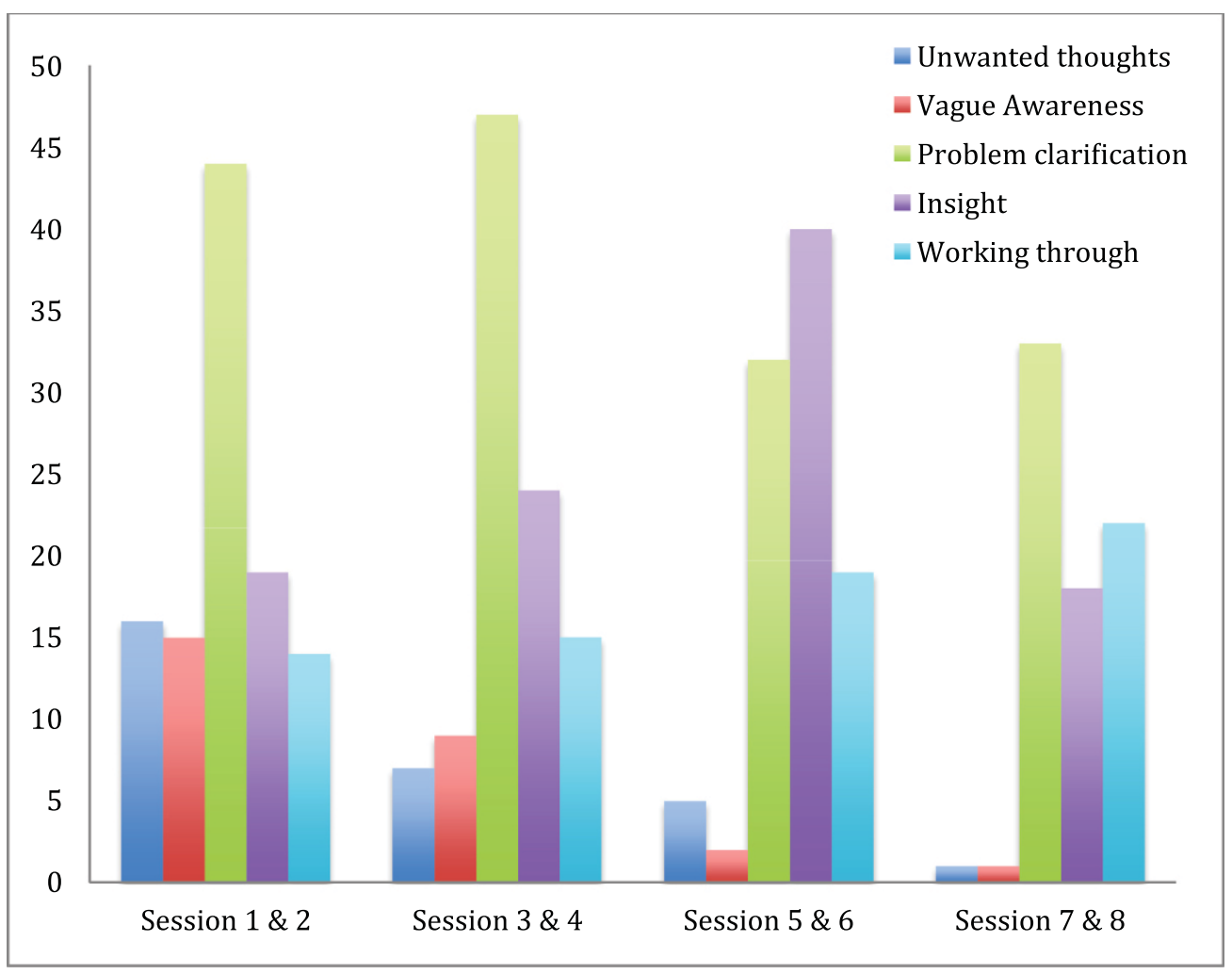

Figure 1. Coding of levels of assimilation across sessions. 
order to determine which elements of the contingency table were significant, we took adjusted $z$-score values for each of the 20 cells and calculated their $p$ values (Beasley \& Schumacker, 1995; Garcia-Perez \& Nunez-Anton, 2003). Z-scores above 1.96 are taken to be significant, but where multiple comparisons are made, as was the case with this analysis, then it is necessary to adjust the level of significance according to the number of cells, which in this case reduced the significance level to $p=0.0025$. The $z$-scores for three cells were significant at this level. Two cells were from sessions 1 and 2 , for level 1, unwanted thoughts $(z=3.3579, p=0.0008)$ and level 2 , vague awareness $(z=3.2768, p=0.001)$ suggesting that the proportion of markers from the early levels of assimilation were significantly higher in the first two sessions, than in later sessions. The third cell where the $z$-score was significant was for level 4 , insight during sessions 5 and $6(z=$ $3.7622, p=0.0002)$.

\section{Study two: changes in therapist verbal behaviour across sessions}

In order to explore potential differences between groups, we used the MAPED ratings to identify groups where was a clear contrast between locations. One pair of groups ( $F$ and $G$ ) both provided sessions from weeks 2 and 8 (demographic details of participants in both locations are provided in Table 4).

There were, however, important differences between participant behaviour in the two sites. In Location $F$, there was a decline in numbers of extracts that were MAPED from 23 in week 2, to 7 in week 8 . Moreover, the modal average rating in both week 2 and week 8 was level 3 (problem clarification), and while there were two working through markers in week 2, there were none in week 8 . Both factors suggest that there was minimal therapeutic change occurring within this location. By contrast, in Location $\mathrm{G}$, the number of overall number of markers increased from 16 in week 2, to 21 in week 8, while the modal marker shifted from level 2 (vague awareness) in week 2 to being jointly level 3 (problem clarification) and level 5 (working through) in week 8.

Table 4. Participant baseline demographic and clinical characteristics.

\begin{tabular}{lcc}
\hline & Location $\mathrm{F}(n=5)$ & Location $\mathrm{G}(n=5)$ \\
\hline Age & 72.2 (range: $73-82)$ & 72 (range: 51-87) \\
Gender & 0 & 2 \\
$\quad$ Male & 5 & 3 \\
$\quad$ Female & & 2 \\
Living status & 4 & 1 \\
$\quad$ Living with spouse & 0 & 1 \\
Living with children & 0 & 1 \\
Living with parent & 1 & \\
Living alone & 23.6 (range: 18-27) & 22.2 (range: 18-30) \\
Mini-Mental State & & 3 \\
Examination (MMSE) score & 3 & 2 \\
Education & 2 & 2 \\
Secondary school & & 2 \\
Further education & 3 & 1 \\
Diagnosis & 2 & 3 \\
Alzheimer's disease & 0 & 2 \\
Vascular dementia & & 2 \\
Mixed dementia & 3 & 3 \\
Taking dementia medication & 2 & \\
Yes & 1 & \\
No & 1 & \\
Taking anti-depressant & 4 & \\
Yes & & \\
No & & \\
\hline
\end{tabular}

\section{Methodology}

In order to examine the role that therapist behaviour in the two locations might play in these differences in participant behaviour, we analysed the therapist verbal behaviour in the extracts using the Hill Therapist Verbal Response Category system (Hill, 1978). This measure has been widely used within psychotherapy process research and classifies therapist or counsellor behaviours into 14 categories.

Ratings of all Location $\mathrm{F}$ verbal behaviours were made by the first and third authors, with the first author and an experienced psychotherapist and clinical psychologist rating therapist behaviours for Location G. Agreement levels were 83\% for Location $\mathrm{F}$ (Kappa $=0.811$; SE $=0.043$; $95 \%$ confidence interval from 0.727 to 0.895 ) and $89 \%$ for $\mathrm{G}$ (Kappa $=0.867$; $\mathrm{SE}=0.036 ; 95 \%$ confidence interval from 0.797 to 0.937 ) both of which can be considered as 'very good'.

\section{Results}

In order to analyse therapist verbal behaviour, we combined the Hill ratings in terms of four categories: minimal response (minimal encourager and approval/reassurance); guidance (information and direct guidance); questions (open and closed questions) and empathic listening (restatement, reflection, non-verbal referent and interpretation) and established a $4 \times 2$ contingency table. It was only possible to analyse therapist behaviour at the two locations for week 2, as there were insufficient behaviours from Location $\mathrm{F}$ for week 8 to permit an analysis.

Analysis of behaviours from week 2 indicated that there was a significant difference between the two locations $\left(\chi^{2}(3)=\right.$ $8.46, p=0.037)$. As Figure 2 indicates, in both groups there was roughly similar use of minimal encouragement and approval reassurance across sessions - both of which are typically short, supportive phrases that either aim to encourage participants to continue talking. Although facilitators asked fewer questions in Location $\mathrm{F}$ in both weeks 2 ( $28 \%$ of behaviours) and $8(26.7 \%)$ compared to Location G (40\% and $43.66 \%$, respectively), post hoc analysis indicated that this was nonsignificant. However, the main difference between the two locations in week 2 was that the facilitators in Location $\mathrm{F}$ used far more information giving and direct guidance behaviours (30\%) than did the facilitators in Location G (7.5\%). Post hoc analysis using an adjusted significance level of 0.0063 indicated that this difference in guidance behaviour was significant $(z=2.754, p=0.0059)$.

\section{Discussion}

Living Well with Dementia groups are intended to help people who are affected by dementia to talk more openly about their illness, and thus to adjust to their diagnosis. Although the Marshall et al. (2015) pilot study was not powered to find statistically significant results, there was a trend towards an increase in levels of self-esteem and quality of life in the intervention group compared to the control condition. As part of the process of developing the intervention, it is important to identify whether there are indeed changes in the way in which participants talked about their dementia, and to examine the influence of therapist verbal behaviour on assimilation. 


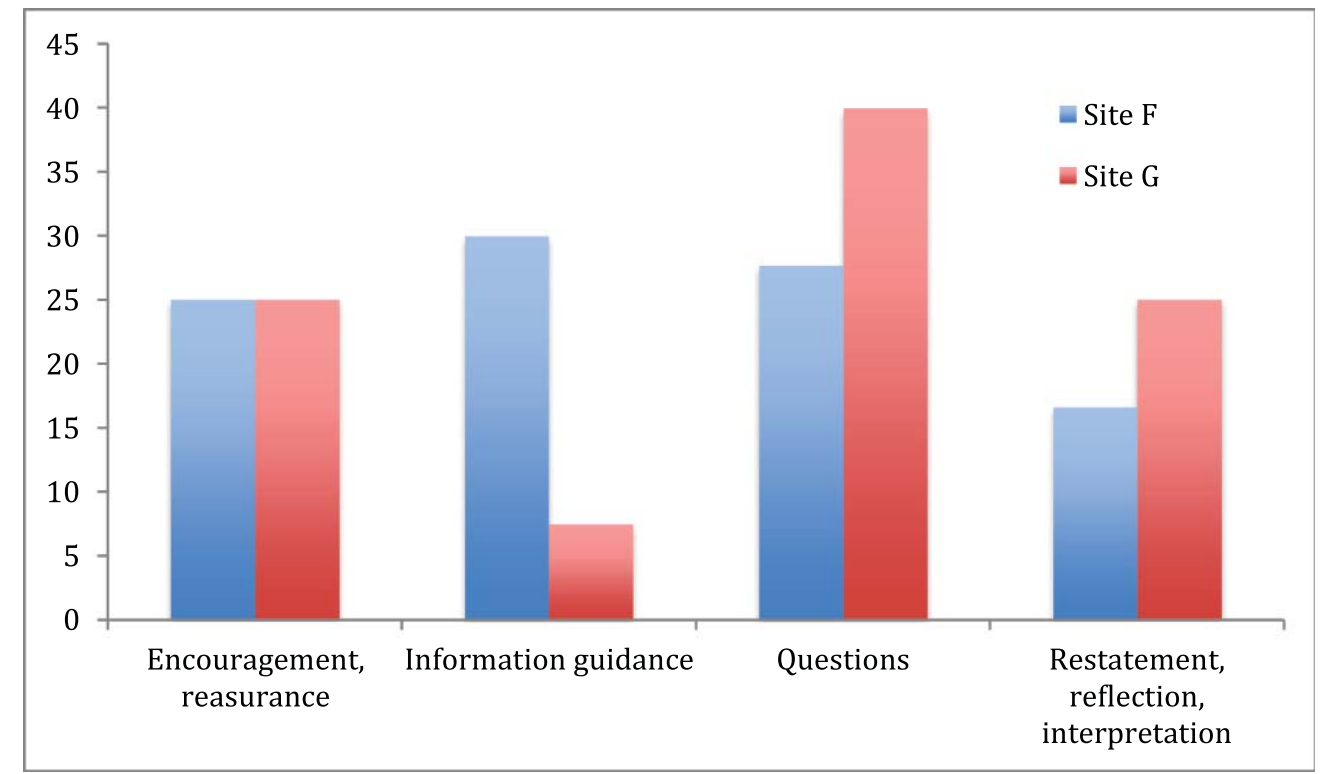

Figure 2. Graph illustrating session 2 percentage of therapist verbal behaviour in Locations $F$ and $G$.

The assimilation model describes awareness as developing through a continuum of eight stages, each of which can be identified by characteristic changes in speech. When a threatening event occurs, for instance a diagnosis of dementia, then according to the model, awareness of that threat is experienced in terms of a dialogue between at least two, contrasting, voices. Typically, a dominant voice will initially express the importance of maintaining the status quo, whilst a problematic voice articulates uncertainty and the need for change. At first, only the dominant voice is heard, and the expression of the problematic experience may be completely warded off, or pushed away. Gradually, however, the problematic and dominant voices combine, so that the problematic experience is fully assimilated into that person's awareness. Movement along this continuum (i.e. from warding off to mastery), is characterised by changes in emotional intensity, so that as the person begins to voice the problematic elements of their experience, so they are likely to become more distressed (see Figure 3).

\section{Changes in participant verbal behaviour}

This study indicates that the discourse of participants in LivDem groups changed significantly over the course of the eight sessions. More specifically, there was a shift after the initial two group sessions away from level 1 and 2 markers,

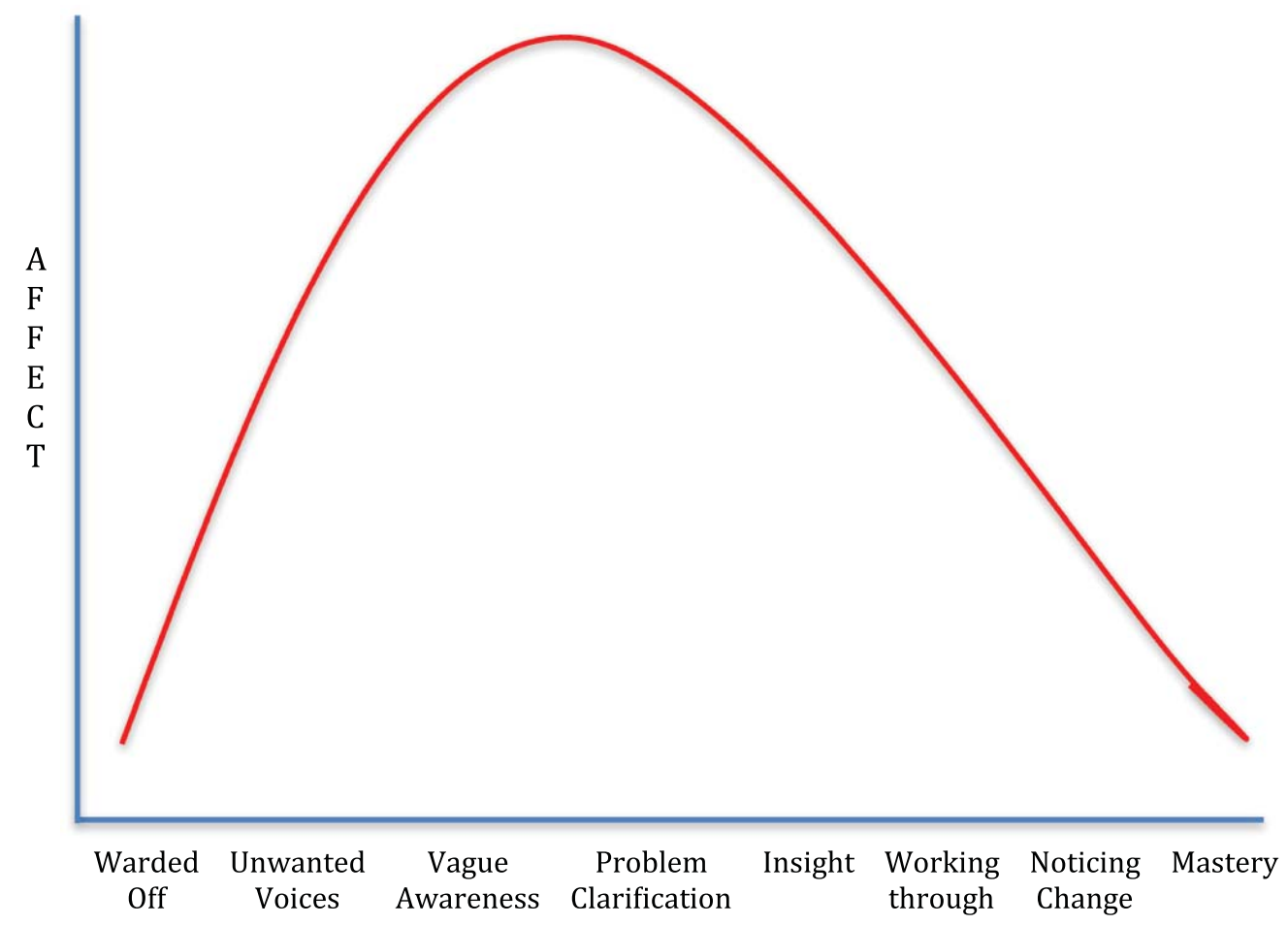

Figure 3. The relationship between levels of assimilation and affect. 
towards markers suggestive of more assimilated levels. In addition, there were significantly more ratings of level 4 (insight) markers in weeks 5 and 6.

The significantly higher number of level 1 and 2 markers in the first two sessions suggests that during this period, there is a tendency towards avoiding engaging with dementia directly. Level 1 and 2 markers are indicative of the person with dementia pushing away knowledge of their dementia. In level 1, the person acknowledges that they experience an aspect of dementia such as memory loss, but avoids discussing or naming their illness directly. Dementia is not specifically identified by name, and is instead, referred to through euphemisms such as 'it', 'the thing that is wrong', or confined to specific, less threatening symptoms. Cheston (2016) has referred to this as the Voldemort phenomena similar to the way in which most characters within the Harry Potter stories are too afraid to directly name the villain, preferring to use terms such as 'he-who-must-not-be-named'. In a level 2 marker, the dementia is still not explicitly acknowledged, but the hallmark of this level is the distress that arises from an internal conflict relating to dementia. The person may describe feeling sadness, angry or worrying, sometimes seeming to be puzzled or confused about what is happening to them, or feeling overwhelmed and that things are getting worse.

Elsewhere, the process of emotionally engaging with the dementia diagnosis has been described as being one of 'oscillating ambivalence' (Robinson, Clare, \& Evans, 2005). This is consistent with the findings of our study: as the group progresses, so the growth of insight, or awareness, is marked not just by an increase in higher levels of assimilation (as indicated by the significantly higher number of level 4 markers in weeks 5 and 6), but also by a lessening of the tendency to push dementia away. The capacity of people with dementia to acknowledge and name their illness is not just an issue of academic interest, as the association between insight, challenging behaviour and caregiver burden has been widely recognised (e.g. Rocca et al., 2010). As one care-giver in the study reported (unpublished thematic analysis):

Well although [insight] seems small is very, very big. I mean once you've accepted you've got a problem then you will accept people trying to help you more. But if you're in denial that you have this problem then of course you're not prepared to accept help from anybody. (Carer. 13, Lines 1227-1231)

\section{Changes in facilitator verbal behaviour}

There were marked differences between MAPED ratings of two locations in week 8: in site $F$, not only were there far fewer extracts that could be MAPED, but a far higher proportion of the extracts (six of the eight) were initiated either by participants rather than by a facilitator. In contrast in site G, in week 8 only 4 of the 23 extracts were initiated by participants. In therapeutic terms, Location $G$ seems to be a healthier site - there are not only more markers in week 8 than in Location $F$, but those that were identified suggest a clear, therapeutic, progression with facilitators being actively involved in helping the group to move on. In Location $\mathrm{F}$, by contrast there are fewer level 1 and 2 markers in week 2, and instead a preponderance of level 3 and 4 markers. We therefore examined whether there were differences in therapist behaviour in the two sites.
The analysis indicates that one difference in therapist behaviour in the two locations is that in Location F, therapists used significantly more information giving and direct guidance behaviour. There was also a non-significant trend towards asking fewer questions. In effect, the analysis suggests that Location $\mathrm{F}$ facilitators tended to say more, and to listen rather less to participants. This may inadvertently have contributed both to the higher frequency of level 3 and 4 markers, and also had the effect of closing down discussions within the group and interfering with the ability of participants to explore the emotional significance of their dementia.

In clinical terms, groups are sometimes said to progress through a series of stages which have been characterised as forming, storming, norming and performing (Tuckman, 1965). In Location F in week 2, the dominant therapist behaviour of information giving and direct guidance may have been more appropriate behaviours for the later stages of the group where the group has become established and participants feel more confident in each other's company. However, at an early stage, therapist behaviours of listening, encouraging and questioning may all have been more appropriate. It is possible, therefore, that the differences in participant assimilation of dementia in week 8 between Locations $F$ and $G$ are associated with the different forms of therapist verbal behaviour in earlier weeks.

The differences between the two locations may be similar to the differences between the impact of psychotherapeutic and psycho-educational groups found by Cheston and Jones (2009). In a small trial, the authors attributed differences in outcome that included increases in depression and anxiety in the psycho-educational arm to the tendency within this condition to talk about too threatening material at too early a stage in group development.

The role of qualitative research approaches within the development of psychotherapeutic approaches to dementia care. The use of mixed methods designs in counselling psychology research has grown in the last two decades (Hanson, Creswell, Clark, Petska, \& Creswell, 2005), as the use of both qualitative and quantitative methodologies in a single body of research may enrich the study findings in ways that using a single methodology could not do. Over the last 30 years, the assimilation model put forward by Stiles and colleagues (e.g. Stiles, 1999, 2001; Stiles et al., 1992) has been used to make sense of those changes that occur during psychotherapy regardless of the therapeutic orientation of therapists. This detailed, qualitative approach complements trials in which psychotherapy is positioned as a quasi-medical intervention that can be assessed through pre- and post-intervention assessment.

The application of Stiles' assimilation model to the discourse of people with dementia has both clinical and theoretical implications for dementia care: markers are clinically important as they identify different opportunities for engaging with people affected by dementia in talking about their illness; they are of theoretical significance because they enable a more nuanced understanding of the psychosocial barriers and facilitators affecting awareness. Thus, the assimilation model suggests that ambivalence plays a central role in the processing of psychologically problematic material: that people can be in two minds about the challenging experiences that affect them. Within a clinical context, then, the initial task within therapy is for people to be able to name their problems as being dementia - something that is only possible if people are supported so that they are able to work through 
their ambivalence at their own pace and without being emotionally overwhelmed (e.g. Cheston, 2013, 2015). Once people are able to talk about the dementia, then they are able to find some emotional distance from the illness. Where therapists behave in a way that does not support this exploration and naming, then this may interfere with the therapeutic process.

In this study, Study 1 provided evidence that there was a process of discursive change in the way in which LivDem participants framed their dementia: in the first two weeks, there was significantly higher use of level 1 and 2 markers indicating that the dementia was being pushed away, or engaged with indirectly. This is consistent with the assimilation model. However, Study 2 suggested that in Location F, an over-reliance by facilitators on information giving and direct guidance in week 2 might be associated with poorer, long-term outcomes in week 8. One interpretation of the results from Study 2 is that the facilitators in Location F did not achieve a therapeutic balance between telling and asking and that consequently, the participants in their group were not able to achieve quite the same changes in discourse between sessions 2 and 8 as shown in Location G. At the same time, we recognise that this is only one of a number of possible explanations, and the potential association between therapist overreliance on telling behaviours in the initial stages of a group and poorer participant outcomes needs to be more rigorously tested in future research.

Conclusion and future directions for research. The role of both individual and group psychotherapy with people with dementia is steadily growing. While much of this research frames evidence as concerning change in pre- and post-intervention outcome measures, it is also important to examine the process of change. Thus, a recent review has concluded that it is important to address other challenges:

\begin{abstract}
... for instance whether the goal of psychotherapy is to improve insight, to reduce anxiety and depression or to reduce challenging behavior and carer stress? Importantly, there is a need to develop a more nuanced understanding of those elements of therapy that helps people affected by dementia to change or, equally relevantly, not to change. (Cheston \& Ivanecka, 2016, p. 27)
\end{abstract}

Helping to understand both the process through which participants become more able to name their illness and at the same time to step back from the emotional pain of the diagnosis, and also the way in which facilitator behaviour augments or detracts from this process is important. As such, this study helps us to understand more about the nature of psychological support, and to adapt the LivDem group intervention to meet the needs of participants.

\section{Notes}

1. Available on request from the first author.

2. http://www.controlled-trials.com/ISRCTN25079950/.

3. REC Number 11/SC/0363, approval dated 18 November 2011, protocol amendments accepted on 28 June 2012 and 23 August 2012.

4. The MAPED coding frame is attached to this paper as a supplementary material or is available on request from the first author.

5. http://graphpad.com/quickcalcs/kappa2/.

\section{Acknowledgments}

We would like to thank all of the participants and their families for participating in this research. The project team would also like to thank the group facilitators (Kathy Chapman-Hill, Jackie Shearing, Lorraine Conduit, Rachel Crane, Angela Lynch, Kate Wilson, Rebecca Burke, Heather
Baldwin, Rachel Woodward, Alison Moren, Sue Ward, Helen Williams, Debbie Bolton, Emma Reed, Susan Ryan and Nicki Short) as well as the project steering group members including Professor Clive Holmes, Elizabeth Bartlett and David Higgenbottom. In addition Lauren Gatting, Andrea Hirons and Saskia Boisevin assisted in the analysis of material.

\section{Disclosure statement}

No potential conflict of interest was reported by the authors.

\section{Funding}

This work was supported by a grant from the National Institute of Health Research, Research for Patient Benefit funding stream [grant number PBPG-0610-22005].

\section{References}

Beasley, T.M., \& Schumacker, R.E. (1995). Multiple regression approach to analyzing contingency tables: Post hoc and planned comparison procedures. The Journal of Experimental Education, 64(1), 79-93.

Cheston, R. (2013). Dementia as a problematic experience: Using the Assimilation Model as a framework for psychotherapeutic work with people with dementia. Neurodisability and Psychotherapy, 1(1), 70-95.

Cheston, R. (2015). The role of the fear-of-loss-of-control marker within the accounts of people affected by dementia about their illness: Implications for psychotherapy. Quaderni di Psicoterapia Cognitiva, 37, 45-66. doi:10.3280/qpc2015-037003

Cheston, R., Gatting, L., Marshall, A., Spreadbury, J., \& Coleman, P. (2015). Markers of assimilation of problematic experiences in dementia within the LIVDEM project. Dementia: the International Journal of Social Research and Policy. (published online September 2, 2015). doi:10.1177/1471301215602473

Cheston, R., \& Howells, L. (2015). A feasibility study of translating "Living Well with Dementia" groups into a primary care IAPT service (innovative practice). Dementia: the International Journal of Social Research and Policy. (published online April 17, 2015). doi:10.1177/1471301215582104

Cheston, R., \& Ivanecka, A. (2016). Individual and group psychotherapy with people affected by dementia: A systematic review of the literature. International Journal of Geriatric Psychiatry. (first published online July 7). doi:10.1002/gps.4529

Cheston, R., \& Jones, R. (2009). A small-scale study comparing the impact of psycho-education and exploratory psychotherapy groups on newcomers to a group for people with dementia. Aging and Mental Health, 13(3), 410-425.

Cheston, R., Jones, K., \& Gilliard, J. (2004). "Falling into a hole": Narrative and emotional change in a psychotherapy group for people with dementia. Dementia: The International Journal of Social Research and Policy, 3(1), 95-103.

Department for Health, Social Services and Public Safety. (2011). Improving dementia services in Northern Ireland: A regional strategy. Belfast: Author.

Department of Health. (2009). Living well with dementia: A national dementia strategy. London: Author.

Garcia-Perez, M.A., \& Nunez-Anton, V. (2003). Cellwise residual analysis in two-way contingency tables. Educational and Psychological Measurement, 63(5), 825-839.

Hanson, W.E., Creswell, J.W., Clark, V.L.P., Petska, K.S., \& Creswell, J.D. (2005). Mixed methods research designs in counseling psychology. Journal of Counseling Psychology, 52(2), 224-235.

Hill, C.E. (1978). Development of a counselor verbal response category system. Journal of Counseling Psychology, 25(5), 461-468.

Honos-Webb, L., Lani, J.A., \& Stiles, W.B. (1999). Discovering markers of assimilation stages: The fear of losing control marker. Journal of Clinical Psychology, 55, 1441-1452.

Honos-Webb, L., \& Stiles, W. (1998). Reformulation of assimilation analysis in terms of voices. Psychotherapy, 35(1), 23-33.

Honos-Webb, L., Surko, M., \& Stiles, W.B. (1998). Manual for rating assimilation in psychotherapy: February 1998 version. Unpublished manuscript. Oxford, $\mathrm{OH}$ : Department of Psychology, Miami University.

Honos-Webb, L., Surko, M., Stiles, W., \& Greenberg, L. (1999). Assimilation of voices in psychotherapy: The case of Jan. Journal of Counselling Psychology, 46, 448-460. 
Lishman, E., Cheston, R., \& Smithson, J. (2016). The paradox of dementia: Meaning making before and after receiving a diagnosis of dementia. Dementia: the International Journal of Social Research and Policy, 15(2), 181-203. (first published online February 2, 2014). doi:10.1177/ 1471301214520781

Logsdon, R., Pike, K.C., McCurry, S.M., Hunter, P., Maher, J., Snyder, L., \& Teri, L. (2010). Early-stage memory loss support groups: Outcomes from a randomized controlled clinical trial. Journal of Gerontology, Series B: Psychological Science and Social Sciences, 65B(6), 691-697.

Marshall, A., Spreadbury, J., Cheston, R., Coleman, P., Ballinger, C. Mullee, M., ... Bartlett, E. (2015). A pilot randomised control trial to compare changes in quality of life for participants with early diagnosis dementia who attend a "Living Well with Dementia" group compared to waiting list control. Aging and Mental Health, 19(6), 526-535. doi:10.1080/ 13607863.2014.954527

Robinson, L., Clare, L., \& Evans, K. (2005). Making sense of dementia and adjusting to loss: Psychological reactions to a diagnosis of dementia in couples. Aging and Mental Health, 9(4), 337-347.

Rocca, P., Leotta, D., Liffredo, C., Mingrone, C., Sigaudo, M., Capellero, B., ... Bogetto, F. (2010). Neuropsychiatric symptoms underlying caregiver stress and insight in Alzheimer's disease. Dementia and Geriatric Cognitive Disorders, 30(1), 57-63.

Scottish Government. (2010). Scotland's national dementia strategy. Edinburgh: Author.
Snow, K., Cheston, R., \& Smart, C. (2015). 'Making sense' of dementia: Exploring the use of the MAPED to understand how couples process a dementia diagnosis. Dementia: The International Journal of Social Research and Policy. (Published online before print, February 18, 2015). doi:10.1177/1471301214564447

Stiles, W. (1999). Signs and voices in psychotherapy. Psychotherapy Research, 9(1), 1-21.

Stiles, W. (2001). Assimilation of problematic experiences. Psychotherapy, 38(4), 462-465.

Stiles, W., Meshot, C., Anderson, T., \& Sloan, W. (1992). Assimilation of problematic experiences: The case of John Jones. Psychotherapy Research, 2, 81-101.

Tuckman, B. (1965). Developmental sequence in small groups. Psychological Bulletin, 63(6), 384-399.

Watkins, B., Cheston, R., Jones, K., \& Gilliard, J. (2006). "Coming out with Alzheimer's disease": Changes in insight during a psychotherapy group for people with dementia. Aging and Mental Health, 10(2), 1-11.

Watts, S., Cheston, R., Moniz-Cook, E., Burley, C., \& Guss, R. (2014). Postdiagnostic support for people living with dementia, in $\mathrm{R}$ Guss et al. (on behalf of the Faculty of the Psychology of Older People, and in collaboration of people living with dementia and the Dementia Workstream Expert Reference Group). Clinical psychology in the early stage dementia care pathway. London: British Psychological Society.

Welsh Government. (2011). National dementia vision for Wales. Cardiff: Author. 